\title{
Non-relativistic metrics from back-reacting fermions
}

\author{
Ling-Yan Hung ${ }^{1 *}$ Dileep P. Jatkar ${ }^{1,2 \dagger}$ and Aninda Sinha ${ }^{1 \ddagger}$ \\ ${ }^{1}$ Perimeter Institute for Theoretical Physics \\ Waterloo, Ontario N2L 2Y5, Canada. \\ ${ }^{2}$ Harish-Chandra Research Institute \\ Chhatnag Road, Jhusi, Allahabad 211019, India.
}

November 6, 2018

\begin{abstract}
It has recently been pointed out that under certain circumstances the back-reaction of charged, massive Dirac fermions causes important modifications to $\mathrm{AdS}_{2}$ spacetimes arising as the near horizon geometry of extremal black holes. In a WKB approximation, the modified geometry becomes a non-relativistic Lifshitz spacetime. In three dimensions, it is known that integrating out charged, massive fermions gives rise to gravitational and Maxwell Chern-Simons terms. We show that Schrödinger (warped $\mathrm{AdS}_{3}$ ) spacetimes exist as solutions to a gravitational and Maxwell Chern-Simons theory with a cosmological constant. Motivated by this, we look for warped $\mathrm{AdS}_{3}$ or Schrödinger metrics as exact solutions to a fully back-reacted theory containing Dirac fermions in three and four dimensions. We work out the dynamical exponent in terms of the fermion mass and generalize this result to arbitrary dimensions.
\end{abstract}

*jhung@perimeterinstitute.ca

†djatkar@perimeterinstitute.ca

†asinha@perimeterinstitute.ca 


\section{Contents}

1 Introduction $\quad 2$

2 Schrödinger spacetimes in Chern-Simons theories 4

3 Schrödinger spacetimes from fermions 5

$3.12+1$ Dimensions . . . . . . . . . . . . . . . . . . . 7

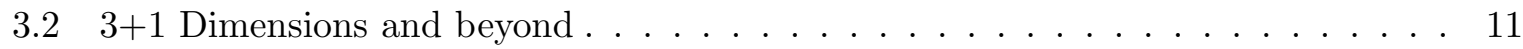

3.2 .1 Massless fermions . . . . . . . . . . . . . . . . 13

3.2 .2 Massive fermions . . . . . . . . . . . . . . . . . 13

3.3 Can there be an exact Lifshitz solution? . . . . . . . . . . . . . . . . 14

4 Discussion $\quad 15$

$\begin{array}{ll}\text { A Conventions } & 17\end{array}$

$\begin{array}{ll}\text { B Spin connection and the Dirac Equation } & 18\end{array}$

C Stress tensor of fermionic probes in AdS 19

C.1 $\mathrm{AdS}_{2} \times R \ldots \ldots \ldots \ldots \ldots \ldots \ldots \ldots$

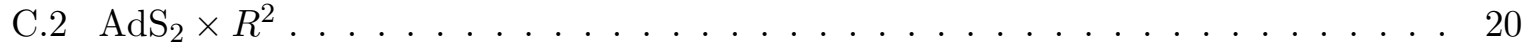

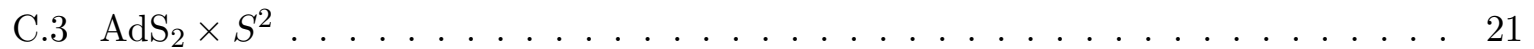

D A toy model justification for our semi-classical treatment 21

\section{Introduction}

In recent times, there has been a concerted effort to find real world applications of the AdS/CFT correspondence. By extending the rules of the correspondence to finite temperature, there are now well established prescriptions to calculate retarded Green functions which enables us to extract transport properties of interesting physical systems. Although none of these systems, strictly speaking, describe the real world, it is hoped that by studying these problems, one will gain intuition about real physical systems which are for example in the same universality class. On one hand, AdS/CFT methods have found applications to particle physics, e.g., the viscosity of the strongly coupled quark gluon plasma [1]. On the other, these methods have been extended to studying table-top condensed matter physics [2]. It is fair to say that applications of gauge/gravity duality will yield many more surprises.

One application that has garnered much attention lately is the computation of fermion Green functions using AdS/CFT which yield non-Fermi liquid type behaviour [3]. In the simplest example, one starts with a Reissner-Nordström AdS black hole and considers the Dirac equation in this background. In the zero temperature limit, the black hole becomes extremal and the near horizon geometry contains an $\mathrm{AdS}_{2}$ factor. This $\mathrm{AdS}_{2}$ is thought to play an important role in the existence of a Fermi surface. The resulting fermion Green function shows non-Fermi liquid type behaviour which is exciting in the context of connections with condensed matter systems, e.g. high $\mathrm{T}_{c}$ superconductivity. However, in the zero temperature limit, the black hole and hence the corresponding dual field theory, still has a large entropy which is far from what is expected in the realistic situations.

Recently in [4], it has been pointed out, that in certain regimes the back reaction of the fermions cannot be ignored. In fact, the $\mathrm{AdS}_{2}$ geometry has been argued to be modified into a 
"Lifshitz" [5] type and the associated entropy enigma is thought to disappear since the horizon area is now zero. Motivated by this, we examine the problem of back-reaction of Dirac fermions in $2+1$ and $3+1$ dimensional gravitational theories in the context of AdS/CFT in some detail. The $2+1$ dimensional bulk theory will describe $1+1$ dimensional field theories. In this context interesting non-Luttinger type behaviour has been found in [6]. The $3+1$ dimensional bulk theory will describe interesting $2+1$ dimensional field theories which are of relevance to condensed matter physics related to high $\mathrm{T}_{c}$ superconductivity. We will restrict our attention to the simplest case where only the metric, a $U(1)$ gauge field and a charged, massive Dirac fermion is involved.

We begin with the discussion of a $2+1$ dimensional bulk theory. It is known that $[7,8,9]$ when one integrates out a charged fermion in $2+1$ dimensions, the effective action that one is led to contains the Maxwell and gravitational Chern-Simons terms. Gravitational ChernSimons theories have been the subject of much attention recently due to the connection with topologically massive gravity (TMG)[10]. In TMG null warped $\mathrm{AdS}_{3}$ have already been found. The new finding in our case is their existence in the presence of $U(1)$ Chern-Simons term $A \wedge F$. We show that in addition to the usual asymptotically $\mathrm{AdS}_{3}$ spacetime, one also gets Schrödinger spacetimes [11] whose dynamical exponent depends on the Maxwell Chern-Simons or the gravitational Chern-Simons terms. Similar solutions (called warped $\mathrm{AdS}_{3}$ ) have already been reported in [12] in the presence of the gravitational Chern-Simons terms. Inspired by this finding, we look for Schrödinger spacetimes as exact solutions to back-reacted Dirac fermions. Since fermions obey the Pauli exclusion principle, they cannot be treated classically. We will treat the system of gravity, gauge field and fermions in a semi-classical manner following [13, 14]. In this approach, the fermion stress tensor and current appearing in the equations of motion are evaluated as expectation values in some state $|Q\rangle^{1}$. We will consider $|Q\rangle$ to be the state made from $N$ fermions. It will turn out that the role of $\hbar$ is played by $1 / N q$ so that for a fixed charge, taking the large $N$ limit will correspond to making $\hbar$ small which is needed for a semi-classical approximation to make sense. The solution to the full problem in a self-consistent manner in general is a very hard task. Typically one resorts to some approximation as in [14] where a WKB approximation is used to derive the Oppenheimer-Volkoff equations. Quite remarkably, our problem will turn out to be amenable to an exact solution! The key ingredient that makes this possible is the fact that the fermion couples to the gauge field. The equations of motion then lead to certain important constraints which make an exact solution possible. In arbitrary bulk dimensions, $D$, we will show that the dynamical exponent is given by

$$
z= \pm 2 m L-(D-2)
$$

where $L$ is the length scale entering in the definition of the cosmological constant in the usual way. Lifshitz metrics are known to emerge as a WKB approximation [4]. It is not known if they are exact solutions to a system of self-gravitating fermions as in the case of the Schrödinger metrics. In the $2+1$ dimensional case, as we will show, Lifshitz metrics are not exact solutions to the equations of motion. However, in higher dimensions we expect there to be more general ways of solving the equations and it could be that other interesting solutions exist. We leave this interesting question as an open problem. ${ }^{2}$

This paper is organized as follows. In section 2, we consider Chern-Simons theories in $2+1$ dimensions and show that Schrödinger type metrics with specific dynamical exponents exist as solutions to the system of equations. Motivated by this, we ask if similar metrics exist as fully back-reacted solutions to Dirac fermions in section 3. We work out the dynamical

\footnotetext{
${ }^{1}$ This method is used quite routinely in nuclear physics. See, e.g., [15].

${ }^{2}$ The fermionic stress tensor eq.(7.43) in [4] can be shown not to lead to Schrödinger spacetimes.
} 
exponent in arbitrary dimensions, i.e., where the original metric would have been $\mathrm{AdS}_{2} \times \mathrm{R}^{D-2}$. We conclude with open questions in section 4. In appendix A we spell out our conventions and in Appendix B, we derive spin connections for a certain class of metrics, which includes, AdS, Schrödinger and Lifshitz metrics and set up the Dirac equation in these backgrounds. In Appendix $\mathrm{C}$ we consider the stress tensor for a Dirac fermion ${ }^{3}$ in a background containing an $\mathrm{AdS}_{2}$ factor. We denote the charge of the fermion by $q$ and mass by $m$. The $\mathrm{AdS}_{2}$ arises as the near horizon geometry of some extremal Reissner-Nordström black hole. In particular, a gauge field $A_{t}$ needs to be turned on for Einstein's equations to be satisfied. We consider a charged, massive Dirac fermion in this background as a probe and work out the stress tensor. We find that quite generally an off-diagonal component of the stress-tensor is turned on. We show that when $m<\sqrt{2 q^{2}+1}$, the back-reaction of fermions becomes important. In appendix $\mathrm{D}$, we study a quantum mechanical toy model in path integral formalism which corresponds to coupling of macroscopic fermi gas to a bosonic harmonic oscillator. We integrate out the fermions and obtain an effective action for the boson. We show that when there are a large number of fermions, the back reaction of fermions is well approximated by replacing fermion number operators by their expectation values evaluated in the $N$ fermion state.

\section{Schrödinger spacetimes in Chern-Simons theories}

We will begin with the discussion of $2+1$ dimensional Einstein-Maxwell-Chern-Simons theory. Our motivation for looking at this theory came from the observation that for a probe fermion in $\mathrm{AdS}_{2}$ geometry, off-diagonal components of the stress tensor are non-zero. (This has been discussed in detail in appendix C.) As a result it may be expected that any non-relativistic modification of the metric will be of the Schrödinger type rather than Lifshitz. As explained in the introduction, on integrating out fermions in $2+1$ dimensions, one gets Maxwell and/or gravitational Chern-Simons theories. Rather than looking at the more complicated problem of back-reaction of fermions, we wish to begin by investigating if Schrödinger type solutions exist for an Maxwell and/or gravitational Chern-Simons theories. Consider the action given in eq. (A.1). The equations of motion for the metric read

$$
R_{\alpha \beta}-\frac{1}{2} g_{\alpha \beta} R-\frac{1}{L^{2}} g_{\alpha \beta}-\frac{1}{2}\left(F_{\alpha \gamma} F_{\beta}^{\gamma}-\frac{1}{4} F^{2} g_{\alpha \beta}\right)+\mu_{G} C_{\alpha \beta}=0,
$$

where $C^{\mu \nu}=\epsilon^{\mu \alpha \beta} \nabla_{\alpha}\left(R_{\beta}^{\nu}-\frac{1}{4} \delta_{\beta}^{\nu} R\right)$ is the Cotton tensor. The equations of motion for the gauge field read

$$
\nabla_{\nu} F^{\nu \mu}+2 \mu_{F} \epsilon^{\mu \nu \rho} F_{\nu \rho}=0 .
$$

It can be shown that the following Schrödinger ${ }^{4}$ (or warped $\mathrm{AdS}_{3}$ [12]) solutions exist

$$
\begin{aligned}
d s^{2} & =-r^{z} d t^{2} \mp 4 r d t d x+\frac{d r^{2}}{4 r^{2}} \\
A_{t} & =\frac{2}{z}\left[r^{z} z(z-1)\left(1 \mp \mu_{G} \pm 2 \mu_{G} z\right)\right]^{1 / 2} \\
z & =0,1, \mp 4 \mu_{F}, \frac{\mu_{G} \mp 1}{2 \mu_{G}}
\end{aligned}
$$

\footnotetext{
${ }^{3}$ Dirac systems in AdS spacetimes have been studied also in [16].

${ }^{4}$ This nomenclature is motivated by the obvious similarity of the metric with the standard Schrödinger spacetimes which involve additional spatial directions. Strictly speaking this is an abuse of nomenclature since in our case there are no spatial directions which transform under dilatations. The conformal boundary of such spacetimes has been discussed in detail in [17].
} 
To avoid clutter, we have set $L=1$. Here $z$ is the standard dynamical exponent and $z=1$ corresponds to the AdS solution, whereas $z=0$ corresponds to a chiral wave AdS solution. The thing to note here is the existence of Schrödinger solutions when either or both of the Chern Simons terms exist. The existence of these solutions in the presence of the gravitational Chern-Simons term can be anticipated from the general discussion in [18]. The case $z=2$, $\left(\mu_{F}=\mp 1 / 2\right.$ or $\left.\mu_{G}=\mp 1 / 3\right)$ is called the null-Warped $\mathrm{AdS}_{3}[12]$.

At this point it is worth noting that Chern-Simons terms are not invariant under parity $(\mathrm{P})$ and time reversal $(\mathrm{T})$ transformations but are invariant under combined transformation PT. This symmetry is also shared by the Schrödinger background, which under $\mathrm{P}$ or $\mathrm{T}$ transformation swaps the sign of $d t d x$ term but the metric is invariant under combined PT transformation. However, in case of $z=1$ the metric is diffeomorphic to AdS metric and preserves $\mathrm{P}$ and $\mathrm{T}$ separately. This does not contradict earlier conclusion because for $z=1$, all components of gauge field vanish and in that case it is natural to expect to recover $\mathrm{P}$ and $\mathrm{T}$ symmetry.

With a bit more work it can also be shown that a Lifshitz metric is not a solution to the above set of equations. We begin with the ansatz

$$
d s^{2}=-\frac{d t^{2}}{r^{2 z}}+\frac{d r^{2}+d x^{2}}{r^{2}}, \quad A_{t}=\phi(r), A_{r}=0, A_{x}=\chi(r)
$$

Then the $t t$ component of the metric equations of motion leads to $r^{2+2 z} \phi^{2}+r^{4} \chi^{\prime 2}=0$ while the $x x$ component leads to $4-4 z^{2}+r^{2+2 z} \phi^{2}+r^{4} \chi^{\prime 2}=0$. Combining these two we are led to $z= \pm 1$. The solution $z=1$ is the usual AdS. The choice $z=-1$ can be shown to lead to imaginary gauge fields. Thus we conclude that Lifshitz is not a solution to this system. We now turn to the more complicated problem of considering back-reaction due to fermions.

\section{Schrödinger spacetimes from fermions}

If we compute the stress tensor for a probe fermion in an $\mathrm{AdS}_{2}$ background as in appendix C, we find that it contains off-diagonal components. This suggests that if their back-reaction is taken into account an exact background geometry of the form of a Lifshitz geometry, which is diagonal, is unlikely. Given however, the intuition from flat space that fermions and the ChernSimons terms are intimately related, and the similarity in structure of the energy-momentum tensors of the gauge fields in the presence of the gauge Chern-Simons term and that of the fermions, one is tempted to conjecture that the back-reaction of charged fermions would also lead to Schrödinger spacetimes, exactly as shown to happen, in the previous section when the back-reaction of gauge fields in the presence of Chern-Simons terms is taken into account. To be explicit, the full Lagrangian we will be considering is (total bulk spacetime dimensions is denoted by $D=d+1$ )

$$
S=\frac{1}{2 \ell_{p}^{d-1}} \int d^{d+1} x \sqrt{-g}\left[R-2 \Lambda-\frac{1}{4} F_{\mu \nu} F^{\mu \nu}+i\left(\bar{\psi} \Gamma^{a} e_{a}^{\mu} D_{\mu} \psi-m \bar{\psi} \psi\right)\right],
$$

where, $\Lambda=-\frac{d(d-1)}{2 L^{2}}$, the $\Gamma$-matrix convention is given in Appendix B. Note here that in general the fermion field will be a sum over modes

$\psi(r, t, x)=\sum_{k}\left(a_{k} \psi_{a, k}(r, t, x)+b_{k}^{\dagger} \psi_{b, k}(r, t, x)\right), \psi^{\dagger}(r, t, x)=\sum_{k}\left(a_{k}^{\dagger} \psi_{a, k}^{\dagger}(r, t, x)+b_{k} \psi_{b, k}^{\dagger}(r, t, x)\right)$

where $a_{k}, a_{k}^{\dagger}, b_{k}, b_{k}^{\dagger}$ denotes the anti-commuting creation and annihilation operators of the corresponding fermion and anti-fermion modes $\psi_{a / b, k}(r, t, x)$ respectively, $k$ denoting some general 
quantum numbers of the modes, each mode $\psi_{a / b, k}(r, t, x)$ a two-component spinor being a solution to the Dirac equation, and that to lowest order ignoring quantum fluctuations of the background we have,

$$
\left\{a_{k_{i}}, a_{k_{j}}^{\dagger}\right\}=\hbar \delta_{k_{i}, k_{j}}=\left\{b_{k_{i}}, b_{k_{j}}^{\dagger}\right\}
$$

while all other anti-commutations between operators vanish. Since the background geometry itself is strongly back-reacted by the fermions, in general it is slightly ambiguous to define what the vacuum would be. We assume the existence of such a stable vacuum such that

$$
a_{k}|0\rangle=b_{k}|0\rangle=0 .
$$

Any general excited state would be of the form

$$
|Q\rangle_{\text {general }}=\prod_{i} a_{k_{i}}^{\dagger} \prod_{j} b_{k_{j}}^{\dagger}|0\rangle,
$$

although they are not generally the $N$-fermion ground state at zero temperature, and are thus not necessarily stable against decay. The Einstein equation is, in our conventions (Appendix A),

$$
R_{\alpha \beta}-\frac{1}{2} g_{\alpha \beta} R-\frac{d(d-1)}{2 L^{2}} g_{\alpha \beta}-\frac{1}{2}\left(F_{\alpha \gamma} F_{\beta}^{\gamma}-\frac{1}{4} F^{2} g_{\alpha \beta}\right)=\frac{1}{2}\left\langle Q\left|T_{\alpha \beta}^{f}\right| Q\right\rangle
$$

where $T^{f}$ is as defined in (C.3) and we reproduce here for convenience

$$
T_{\mu \nu}^{f}=\frac{1}{2}\left(-i \bar{\psi} \Gamma_{(\mu} D_{\nu)} \psi+i \bar{\psi} \overleftarrow{D}_{(\mu} \Gamma_{\nu)} \psi\right)
$$

This form of the stress tensor is given in [19] and to the best of our knowledge was first given in [13]. The covariant derivative $D_{\mu}$ is defined in equation (C.2). The Maxwell's equations are given by

$$
\nabla^{\nu} F_{\mu \nu}=\left\langle Q\left|j_{\mu}\right| Q\right\rangle \quad j_{\mu}=-q \bar{\psi} \Gamma_{\mu} \psi
$$

Note that in the above expressions, the fermion stress tensor and electric currents that back-react on the background are really the expectation values of the corresponding operators, evaluated on the state $|Q\rangle$. At zero temperature, the ground state of $N$ fermions would correspond to piling up the various modes, starting from the bottom mode. Naively, both the currents and the fermion stress tensor would take roughly the form $\sum_{k} a_{k}^{\dagger} a_{k}\left|\psi_{k}(r, t, x)\right|^{2}$, whose expectation value in the state $|Q\rangle$, would involve a sum over contributions of modes that are occupied in $|Q\rangle$. However, here we should note that in general one is confronted with a diverging expectation value due to contributions even of unfilled modes. It is ambiguous to define normal-ordered operators in curved space, since vacuum energy gravitates as well [19]. Here we approach the problem via analytic continuation [19]. As we will see in the following section, the modes that are consistent with the background symmetry and the Gauss's law constraints are simply chiral plane waves along $t$ in Schrödinger space, with trivial radial dependence. Therefore they contribute in the expectation value of the current or stress tensor via $\left\langle Q\left|\sum_{i} a_{i}^{\dagger} a_{i}\right| Q\right\rangle$ simply as $\sum_{i}^{N}(1)=N$ and thus precisely in the same way the unoccupied anti-fermion modes, which we have infinitely many of them will contribute via

$$
\left\langle Q\left|\sum_{i} b_{i} b_{i}^{\dagger}\right| Q\right\rangle=1+1+1 \ldots=\zeta(0)=-\frac{1}{2},
$$

where in the second equality we attempt to regularize it via analytic continuation. Adopting this approach would correspond to shifting $N$ by $-\frac{1}{2}$. Note that this is sub-leading in $N$, and in the large $N$ limit, which is the main focus of this paper, we will ignore this term.

The analysis that we present below is very similar in spirit to that in [14] for a system of self-gravitating fermions. The essential steps in this are as follows: 
1. We want to find a particular set of fields involving the metric, gauge field and fermions such that it is an exact solution to the equations of motion given above.

2. In [14], a certain ansatz for the metric compatible with some pre-supposed symmetries is taken. The Dirac equation is solved in this background for a complete set of modes.

3. With these solutions, one works out the stress tensor $T^{f}$ entering Einstein's equations, taking care of the antisymmetric nature of the fermions.

4. The full set of equations is now solved self consistently.

In the regime of large quantum numbers and using a WKB approximation [14] recovers the Oppenheimer-Volkoff equations ${ }^{5}$. In [4], a WKB approximation was used to obtain a Lifshitz solution. We will not make any such approximations in what we do. In addition to the metric and fermions as in [14] we also have a gauge field which makes the calculation somewhat more complicated. To keep life simple, we will begin with the ansatz for a Schrödinger spacetime with an undetermined dynamical exponent. Then we will ask if there exist self-consistent set of solutions. To be compatible with the symmetries of the problem, we will assume that the gauge field is only dependent on the radial coordinate. At this stage, we note that the stress tensor for fermions has an $\hbar$ [14]. We will find that $1 / N q$ plays the role of $\hbar$ in what we do. A quick way to see that this is true is that in our solution, the stress tensor from fermions is of the same order as that of the Maxwell fields and the Einstein tensor, not surprisingly since we found a fully back-reacted solution. However, the general form of the charged fermion stress tensor is roughly $T^{f} \sim \hbar N q$, using the anti-commutation relation of the operators. Therefore our solution naturally forces $N q \sim 1 / \hbar$. It is interesting to note here that for small $N q$, it would imply that $\hbar$ is large and that a classical approximation would not stand. This conforms with our intuition that a classical approximation improves when the fermi gas becomes macroscopic. To find further support for our approach, we present a path-integral calculation of a toy-model consisting of fermions coupled to bosons in Appendix D. We found that quite generically in the limit of large fermion number $N$, our semi-classical treatment adopted here is a good approximation.

\section{$3.1 \quad 2+1$ Dimensions}

Like the Chern-Simons terms, massive fermions in $2+1$ dimensions also break $\mathrm{P}$ and $\mathrm{T}$ symmetry. We therefore expect the result here to be similar to what we got in the presence of Chern-Simons terms. Anticipating that the back-reacted exact geometry is going to be given by the Schrödinger metric, we start with the ansatz metric exactly as in the previous section, namely

$$
d s^{2}=L^{2}\left(-r^{z} d t^{2}-4 \epsilon r d t d x+\frac{d r^{2}}{4 r^{2}}\right)
$$

where $\epsilon= \pm 1$, and leave the gauge fields and the index $z$ to be determined by the equations of motion. The three sets of equations involved are the Maxwell equations, the Einstein equations and the Dirac equations, to be solved simultaneously. We will, unless otherwise specified, set $L=1$ to avoid clutter in the equations.

To begin with, we compute the spin-connection necessary to build the Dirac equations. Choosing explicitly the vierbein as

$$
e^{0}=r^{-\frac{z}{2}}\left(r^{z} d t+2 \epsilon r d x\right), \quad e^{1}=2 \epsilon r^{1-\frac{z}{2}} d x, \quad e^{d}=\frac{1}{2 r} d r
$$

\footnotetext{
${ }^{5}$ In [20], a similar analysis was used to study holographic neutron stars.
} 
the corresponding spin-connection is

$$
\omega^{01}=\frac{z-1}{2} d r, \quad \omega^{0 d}=r^{-\frac{z}{2}}\left(r^{z} z d t+2 \epsilon r d x\right), \quad \omega^{1 d}=r^{-\frac{z}{2}}\left(r^{z}(z-1) d t+2 \epsilon r d x\right) .
$$

Considering a particular mode of the Dirac spinor,

$$
\psi_{\omega, k}(r, t, x)=e^{-i \omega t-i k x}\left(\begin{array}{c}
\psi_{+, \omega, k}(r) \\
\psi_{-, \omega, k}(r)
\end{array}\right),
$$

the Dirac equation for a fermion of mass $m$ takes the form

$$
\begin{aligned}
& -\left(2+4 i q r A_{r}(r)\right) \psi_{-, \omega, k}(r)+2 i r^{-z / 2}\left(\omega-q A_{t}(r)\right)\left\{\psi_{-, \omega, k}(r)-\psi_{+, \omega, k}(r)\right\} \\
& +\left[1+2 m-z+i r^{(-1+z / 2)}\left(k-q A_{x}(r)\right)\right] \psi_{+, \omega, k}(r)-4 r \psi_{-, \omega, k}^{\prime}(r)=0, \\
& -2 i r^{-z / 2}\left(\omega-q A_{t}(r)\right)\left\{\psi_{-, \omega, k}(r)-\psi_{+, \omega, k}(r)\right\}+\left(2+4 i q r A_{r}(r)\right) \psi_{+, \omega, k}(r) \\
& -\left[1+2 m-z-i r^{(-1+z / 2)}\left(k-q A_{x}(r)\right)\right] \psi_{-, \omega, k}(r)+4 r \psi_{+, \omega, k}^{\prime}(r)=0 .
\end{aligned}
$$

It is simplest to start examining the Maxwell's equations (3.8). We shall begin by picking the gauge

$$
A_{r}=0 .
$$

We will be looking at radially symmetric solution such that the metric and the gauge fields contain only $r$ dependence. Given our gauge choice, the Maxwell's equation for the $A_{r}$ component becomes purely a constraint on the vanishing of the $r$-component of the fermion current. That gives, explicitly,

$$
j_{r}=-q \epsilon \frac{\left(\hat{\psi}_{-}^{\dagger} \hat{\psi}_{-}-\hat{\psi}_{+}^{\dagger} \hat{\psi}_{+}\right)}{2 r}
$$

where we have defined the operators $\hat{\psi}_{ \pm}$, to be distinguished from the modes $\psi_{ \pm, \omega, k}$

$$
\psi=\left(\begin{array}{c}
\hat{\psi}_{+} \\
\hat{\psi}_{-}
\end{array}\right), \quad \hat{\psi}_{ \pm}=\sum_{i} a_{i} e^{-i\left(\omega_{i} t-k x\right)} \psi_{ \pm, \omega, k}^{a}+b_{i}^{\dagger} e^{i\left(\omega_{i} t-k x\right)} \psi_{ \pm, \omega, k}^{b} .
$$

At the same time, to preserve the symmetry of the system, we would like to keep the magnetic field zero i.e. $F_{x r}=0$, we allow only for

$$
A_{x}(r)=a_{1}^{x}
$$

for some constant $a_{1}^{x}$, which, from the gauge equations, further give

$$
A_{x}^{\prime}(r)+r A_{x}^{\prime \prime}(r)=-q \epsilon\left(\left(r^{-z / 2}\right)\langle Q|\left(\hat{\psi}_{-}^{\dagger}-\hat{\psi}_{+}^{\dagger}\right)\left(\hat{\psi}_{-}-\hat{\psi}_{+}\right)\right)|Q\rangle=0 .
$$

To satisfy both requirements $(3.17,3.20)$, in general we have

$$
\psi_{-, \omega, k}^{a}(r)=\psi_{+, \omega, k}^{a}(r), \quad \psi_{-, \omega, k}^{b}(r)=\psi_{+, \omega, k}^{b}(r),
$$

for each contributing mode occupied in state vector $|Q\rangle$.

Now going back to the Dirac equations, given the relation (3.21) the Dirac equations reduce to

$$
r\left((1-2 m+z) \psi_{+, \omega, k}^{a, b}+4 r \psi_{+, \omega, k}^{a, b}{ }^{\prime}\right) \pm i r^{\frac{z}{2}}\left(k-q A_{x}\right) \psi_{+, \omega, k}^{a, b}=0 .
$$

It is important to note that with the relation (3.21), the $A_{t}$ and time-derivatives have completely dropped out from the Dirac equation. This implies that all the modes with different $\omega$ share the same radial wave-function, which also means

$$
\psi_{ \pm, \omega, k}^{a}=\psi_{ \pm, \omega, k}^{b}
$$


Also, the term proportional to $\left(k-q A_{x}\right)$ renders the two equations inconsistent. To obtain non-trivial solutions, it implies

$$
a_{1}^{x}=\frac{k}{q}
$$

which cannot be satisfied generally for an arbitrary $k$-mode in a given background of some $a_{1}^{x}$. This implies that the modes consistent with the background are those with arbitrary $\omega$ but fixed $k$ such that (3.24) is satisfied. These solutions however are gauge equivalent to having $k=A_{x}=0$, via the gauge transformation $\chi=-\frac{k}{q} x$ i.e. $\psi \rightarrow e^{-i k x} \psi$, such that correspondingly $A_{x} \rightarrow A_{x}-\frac{k}{q}$. We will thus from now on set $A_{x}=k=0$. i.e., in full generality, we have

$$
\psi(t, r)=\left(\begin{array}{l}
1 \\
1
\end{array}\right) \sum_{i}\left(a_{\omega_{i}} e^{-i \omega_{i} t} \Psi_{+}(r)+b_{\omega_{i}}^{\dagger} e^{i \omega_{i} t} \Psi_{+}(r)\right),
$$

where we have

$$
\psi_{ \pm, \omega, 0}^{a}=\psi_{ \pm, \omega, 0}^{b}=\Psi_{+}(r) .
$$

To avoid complications, for the moment we treat the spectrum as discrete. This does not alter our results in any significant way even when the continuous limit is taken. We would like to consider the back-reaction of a large number of fermions. This can be done by building explicitly a fermi gas by constructing the $N$-fermion state vector $|Q\rangle$

$$
|Q\rangle=\prod_{i}^{N} a_{\omega_{i}}^{\dagger}|0\rangle
$$

Let us emphasize here that $N$ is the number of modes excited, which is infinity in a continuum limit for finite fermi-energy. The fermion density distribution $\rho$ however is smooth. As we have demonstrated above, since modes of different $\omega$ have the same $r$-wave function, evaluating the expectation value of the stress tensor and the current on $|Q\rangle$, would be proportional to the term, schematically given by

$$
\left\langle Q\left|\hat{\psi}_{+}^{\dagger} \hat{\psi}_{+}\right| Q\right\rangle=\left|\Psi_{+}(r)\right|^{2}\left\langle Q\left|\sum_{i}^{N} a_{\omega_{i}}^{\dagger} a_{\omega_{i}}+\sum_{i}^{\infty} b_{\omega_{i}}^{\dagger} b_{\omega_{i}}\right| Q\right\rangle=\hbar\left(N-\frac{1}{2}\right)\left|\Psi_{+}(r)\right|^{2}
$$

where the unfilled-anti-fermionic states contribution $b b^{\dagger}$ has been regularized as discussed in (3.9). Since they are sub-leading in $N$ after regularization, we will not include them explicitly in the rest of our discussion. Note that in the expectation values of the operators, cross terms occurring in the product $\hat{\psi}^{\dagger} \hat{\psi}$ vanishes, and as a result both the stress tensor and the currents have no $x, t$ dependence. Returning to the Dirac equation, one can then readily solve it to give

$$
\Psi_{+}(r)=p_{1} r^{\delta_{p}}, \quad \delta_{p}=\frac{1}{4}(-1+2 m-z),
$$

for some suitable overall constant normalization $p_{1}{ }^{6}$. The Maxwell equations is left with one component determining $A_{t}$. The expectation of the $t$-component of the current is given by

$$
\left\langle Q\left|j_{t}\right| Q\right\rangle=-q r^{\frac{z}{2}}\left\langle Q\left|\left(\left|\hat{\psi}_{-}\right|^{2}+\left|\hat{\psi}_{+}\right|^{2}\right)\right| Q\right\rangle=-2 r^{\frac{z}{2}+2 \delta_{p}} N q \hbar\left|p_{1}\right|^{2} .
$$

\footnotetext{
${ }^{6}$ Note that because of the way we defined the measure of the frequency sum in (3.25), the normalization $p_{1}$ should be $\omega$ independent to be consistent with the anti-commutation relations (3.3) and subsequently canonical anti-commutation relation between the $\psi$ and $\bar{\psi}$.
} 
Substituting in the Maxwell equations we have

$$
\hbar N\left|p_{1}\right|^{2} q r^{\frac{1}{2}\left(z+4 \delta_{p}\right)}-2 r A_{t}^{\prime}-2 r^{2} A_{t}^{\prime \prime}=0,
$$

which gives,

$$
A_{t}=\hbar \frac{2 N\left|p_{1}\right|^{2} q r^{\frac{1}{2}\left(z+4 \delta_{p}\right)}}{\left(z+4 \delta_{p}\right)^{2}}+c_{2}^{t}+c_{1}^{t} \log r,
$$

for some constants $c_{i}^{t}$. The log term would appear in the energy-momentum tensor as a lone term. We therefore set

$$
c_{1}^{t}=0
$$

Returning to the Einstein equations, we inspect specifically the $t t$ component. Evaluating the LHS of eq. (3.6), we get

$$
R_{t t}-\frac{1}{2} g_{t t} R-g_{t t}-\frac{1}{2}\left(F_{t \gamma} F_{t}^{\gamma}-\frac{1}{4} F^{2} g_{t t}\right)=2\left(r^{z}(-1+z) z-r^{2} A_{t}^{\prime 2}\right),
$$

and the expectation value of the $t t$-component of the fermionic energy-momentum tensor evaluated on the state $|Q\rangle$,

$$
\begin{aligned}
\left\langle Q\left|T_{t t}^{f}\right| Q\right\rangle & =-2 r^{\frac{z}{2}}\left(\left\langle Q\left|\hat{\psi}_{+} \partial_{t} \hat{\psi}_{+}\right| Q\right\rangle-q A_{t}\left\langle Q\left|\hat{\psi}_{+} \hat{\psi}_{+}\right| Q\right\rangle\right) \\
& =-2 r^{\frac{z}{2}+2 \delta_{p}} \hbar\left(\sum_{i}^{N}\left|p_{1}\right|^{2} \omega_{i}-q A_{t} \sum_{i}^{N}\left|p_{1}\right|^{2}\right) \\
& =-2 r^{\frac{z}{2}+2 \delta_{p}}\left|p_{1}\right|^{2} \hbar\left(\sum_{i}^{N} \omega_{i}-q N\left(c_{2}^{t}-\hbar \frac{2 N\left|p_{1}\right|^{2} q r^{\frac{1}{2}\left(z+4 \delta_{p}\right)}}{\left(z+4 \delta_{p}\right)^{2}}\right)\right),
\end{aligned}
$$

which, together with (3.32), are substituted in to the Einstein equation (3.6). The $t$-component of the equation is thus left with three different terms. The metric contributes a term that goes like $r^{z}$, and the gauge and fermion stress tensor contain two different terms: one contributes to a $r^{z+4 \delta_{p}}$, and another term proportional to $q N c_{2}^{t}-\sum_{i}^{N} \omega_{i}$ goes like $\sim r^{\frac{z}{2}+2 \delta_{p}}$. The only way to obtain a non-trivial solution is to switch this latter term off. Therefore we have,

$$
c_{2}^{t}=\frac{\sum_{i}^{N} \omega_{i}}{q N}=\sum_{n=0}^{N-1} \frac{n \pi}{N q l}=\frac{(N-1) N \pi}{2 q N l} \sim \frac{\omega_{F}}{2 q},
$$

where $l$ is the regulated size of the time direction. To obtain non-vanishing spinor solution, one is forced to take $\delta_{p}=0$. This relates the index $z$ to the fermion mass,

$$
z=2 m L-1
$$

where we have restored $L$. With that the Einstein equation is thus reduced simply to an algebraic equation constraining $p_{1}$

$$
-2 N^{2} \hbar^{2}\left|p_{1}\right|^{4} q^{2}+(z-1) z^{3}=0,
$$

which readily yields

$$
\left|p_{1}\right|^{2}=\frac{\left((m L-1)(2 m L-1)^{3}\right)^{\frac{1}{2}}}{\hbar N q},
$$

where we have restored the radius $L$ here. However, $p_{1}$ is the normalization of our plane wave solutions. It has to take a fixed value. The normalization of the wave function is determined by

$$
\int d^{3} x \Psi_{+}^{\dagger}(r) \Psi_{+}(r)=\left|p_{1}\right|^{2} V=1,
$$


where $V$ is the regulated volume.

Therefore, the relation (3.39) fixes the value of $N$ that would back-react to give rise to the particular geometry and electric fields.i.e.

$$
\frac{\left((m L-1)(2 m L-1)^{3}\right)^{\frac{1}{2}}}{\hbar N q}=\frac{1}{V},
$$

which gives

$$
\frac{N q \hbar}{V}=\rho \hbar=\left((m L-1)(2 m L-1)^{3}\right)^{\frac{1}{2}} \sim \mathcal{O}(1),
$$

where $\rho$ is the charge density. Einstein equation dictates that $\hbar \rho \sim 1$ which is expected from our intuitive argument presented at the beginning of the section.

Being a constant solution, the $t r$-component of the fermion energy momentum tensor

$$
T_{t r}^{f}=\frac{i r^{z}}{2}\left(\hat{\psi}_{+}^{\dagger} \hat{\psi}_{+}-\hat{\psi}_{+}^{\dagger} \hat{\psi}_{+}^{\prime}\right)
$$

which is a lone term in the Einstein equation also vanishes. Similarly replacing $m \rightarrow-m$ we will get $z=-2 m L-1$.

There are a few interesting limits to take. One could for example consider taking the limit $m L \rightarrow \frac{1}{2}$ such that $z \rightarrow 0$. Staring at $(3.38,3.39)$ it means a non-trivial fermion solution is only recovered for $q \rightarrow 0$ at the same rate. In this limit however the gauge component $A_{t}$ becomes a constant, but in fact an infinite constant since $c_{2}^{t}$ is proportional to $1 / q$. Remarkably this is precisely what happens in the gauge-gravitational Chern-Simons analysis when we take the limit $z \rightarrow 0$ and $\mu_{F} \rightarrow 0$ in equation (2.5). Another possibility to consider is that for $q=0$ to start with, then the homogenous solution in the Maxwell's equations, namely the log term, could have been allowed without entering into Einstein equation through the fermion stress tensor. Indeed we checked that this is a solution, provided that $m=\frac{1}{2}$ and $z=0,1$. When $z=1$ the fermion radial wave-function is in fact not a constant but goes like

$$
\Psi_{+}(r) \sim r^{-1}
$$

When one compares with the gauge-gravitational Chern-Simons setting, where one analogously takes $\mu_{F}=0$ and allows for the homogenous solution $A_{t} \sim c_{2}^{t}+c_{1}^{t} \log r$, it turns out $z=0,1$ are again solutions, provided that one has to take

$$
\mu_{G}(z-1) z \sim c_{1}^{t^{2}}
$$

in either case. i.e. $\mu_{G}$ curiously approaches infinity. There appears to be a one-to-one correspondence between the gauge-gravitational Chern-Simons theory and the Einstein-MaxwellDirac system, as would be expected if Chern-Simons terms should be recovered by integrating out fermions. Although our results suggest that the relationship between the value of the Chern-Simons couplings and the fermion mass and charge depart from that in flat space.

\section{$3.23+1$ Dimensions and beyond}

The procedure above can be simply generalized to higher dimensions. Consider for concreteness the case $D=3+1$. In this case the metric ansatz we use is the 4 -d Schrödinger metric,

$$
d s^{2}=L^{2}\left(-r^{z} d t^{2}-4 \epsilon r d t d x+r d y^{2}+\frac{d r^{2}}{4 r^{2}}\right)
$$


where we will again set $L=1$ from now on. Using the same choice of vierbeins as in $2+1$ with the addition of

$$
e^{3}=\sqrt{r} d y
$$

where the hat denotes tangent coordinates. The spin-connections will be given again by (3.12), with only the new addition of

$$
\omega^{d 3}=-\sqrt{r} d y
$$

Choosing again the Dirac spinor to be

$$
\psi=\sum_{i, \chi} e^{-i\left(\omega t-k_{1} x-k_{2} y\right)} a_{\omega, k_{1}, k_{2}}^{\chi} \psi_{\omega, k_{1}, k_{2}}^{\chi}+e^{i\left(\omega t-k_{1} x-k_{2} y\right)} b_{\omega, k_{1}, k_{2}}^{\chi \dagger} \psi_{\omega, k_{1}, k_{2}}^{\chi},
$$

where $\chi=\{1,2,3,4\}$ are spinor polarization indices,

$$
\psi_{\omega, k_{1}, k_{2}}^{\chi}=\left(\begin{array}{c}
\psi_{+, \omega, k_{1}, k_{2}}^{\chi}(r) \\
\psi_{-, \omega, k_{1}, k_{2}}^{\chi}(r)
\end{array}\right), \quad \psi_{ \pm, \omega, k_{1}, k_{2}}^{\chi}=\left(\begin{array}{c}
\psi_{ \pm, \omega, k_{1}, k_{2}}^{\chi, 1} \\
\psi_{ \pm, \omega, k_{1}, k_{2}}^{\chi, 2}
\end{array}\right),
$$

and we take as before $k_{i}=0, i=1,2$. For clear notations, we will omit the polarization index $\chi$ in the following, only to look explicitly for the non-trivial spinor polarization that would solve the system of equations. Assuming only $r$ dependence and going through similar analysis as in the previous section leads to very simple results. Starting again with the Maxwell equations to obtain constraints on the spinors, the equation for $A_{r}$ gives

$$
\hat{\psi}^{2}{ }_{+}^{\dagger} \hat{\psi}^{1}{ }_{-}+\hat{\psi}^{1}{ }_{+}^{\dagger} \hat{\psi}_{-}^{2}-\hat{\psi}^{1}{ }_{-}^{\dagger} \hat{\psi}^{2}{ }_{+}-\hat{\psi}^{1}{ }_{-}^{\dagger} \hat{\psi}_{+}^{2}=0
$$

where as in the previous section we are again denoting the components of the $\psi$ field operator with a hat, to be distinguished from the modes. The simplest solution is again taking, for each mode

$$
\psi_{+, \omega, k_{1}, k_{2}}^{2}=\psi_{-, \omega, k_{1}, k_{2}}^{2}=0 .
$$

This choice also easily ensures the vanishing of the source currents for $A_{x}$ and $A_{y}$. Subsequently the Einstein equation and the Maxwell equations would again imply

$$
A_{r}=A_{x}=A_{y}=0 \text {. }
$$

Fermions taking constant value is forced to be a solution

$$
\psi_{ \pm, \omega, k_{1}, k_{2}}^{1}=p_{ \pm}^{1},
$$

for some normalization constants $p_{ \pm}^{1}$, and we arrive at

$$
A_{t}=c_{1}^{t} \log r+c_{2}^{t}+\left\langle Q\left|\frac{q\left(\hat{\psi}_{-}^{1 \dagger} \hat{\psi}_{-}^{1}+\hat{\psi}_{+}^{1 \dagger} \hat{\psi}_{+}^{1}\right) r^{\frac{z}{2}}}{z(1+z)}\right| Q\right\rangle,
$$

where

$$
|Q\rangle=\prod_{i}^{N_{+}} a_{+, \omega_{i}}^{\dagger} \prod_{i}^{N_{-}} a_{-, \omega_{i}}^{\dagger}|0\rangle, \quad\left\langle Q\left|\hat{\psi}_{ \pm}^{1 \dagger} \hat{\psi}_{ \pm}^{1}\right| Q\right\rangle=N_{ \pm}\left|p_{ \pm}^{1}\right|^{2},
$$

and $a_{ \pm, \omega_{i}}^{\dagger}$ denotes the creation operators of the fermion modes $\psi_{ \pm, \omega_{i}}^{1}$ respectively. $N_{ \pm}$are the fermion numbers for the \pm modes respectively. As in $d=2+1$, Einstein equation dictates that $c_{1}^{t}=0$ and

$$
\left(N_{+}\left|p_{+}^{1}\right|^{2}+N_{-}\left|p_{-}^{1}\right|^{2}\right) c_{2}^{t}=\frac{1}{q}\left(\sum_{i} \omega_{+, i}\left|p_{+}^{1}\right|^{2}+\sum_{j} \omega_{-, j}\left|p_{-}^{1}\right|^{2}\right) .
$$


The two remaining Dirac equations for constant fermions cannot be satisfied simultaneously for arbitrary massive fermions when both $\psi_{+}^{1}$ and $\psi_{-}^{1}$ are non-vanishing. Explicitly, the remaining Dirac equations are given by

$$
p_{-}^{1}(2+2 m+z)=0, \quad p_{+}^{1}(2-2 m+z)=0 .
$$

We are thus left with three different possibilities.

\subsubsection{Massless fermions}

For massless fermions,

$$
z=-2
$$

and the remaining Einstein equation is

$$
\frac{3\left(12-\langle Q|\left(\hat{\psi}_{-}^{1 \dagger} \hat{\psi}_{-}^{1}+\hat{\psi}_{+}^{1 \dagger} \hat{\psi}_{+}^{1}\right)^{2} q^{2}\right)|Q\rangle}{2 r^{2}}=0
$$

which relates $N_{-}$to $N_{+}$.

\subsubsection{Massive fermions}

For massive fermions, there are two independent sets of solutions.

$$
p_{+}^{1}=0, \quad N_{-}\left|p_{-}^{1}\right|^{2}= \pm \frac{2 \sqrt{\left(9+18 m+8 m^{2}\right)(m+1)(2 m+1)^{2}}}{q \sqrt{4 m+3}},
$$

and

$$
z=2 m L-2 .
$$

This solution applies when $m \neq-\frac{3}{4}$. When $m$ actually takes that value, $\psi_{-}^{1}$ becomes unconstrained because the Einstein equation vanishes without further constraint on $\psi_{-}^{1}$. Similarly one could have

$$
p_{-}^{1}=0, \quad N_{+}\left|p_{+}^{1}\right|^{2}= \pm \frac{2 \sqrt{\left(9-18 m+8 m^{2}\right)(m-1)(2 m-1)^{2}}}{q \sqrt{4 m-3}}
$$

where

$$
z=-2 m L-2
$$

and $m \neq \frac{3}{4}$. Otherwise $N_{+}$is unconstrained.

Here we have an interesting pattern to note. When $D=2+1$, the resultant index $z=2 m L-1$ and at $D=3+1$ that gives, depending on chirality, $\pm 2 m L-2$. It turns out that exact solutions of charged constant spinor solutions and Schrödinger metrics can be found in arbitrary dimensions. In $D=4+1$ for example we have

$$
z= \pm 2 m L-3 .
$$

There is a clear pattern in the constraint on $z$ as we go to higher dimension, namely

$$
z= \pm 2 m L-(D-2) .
$$

The reason it gets shifted in this particular manner is that these constraints follow from requiring the coefficient multiplying $\psi$ in the Dirac equation to vanish, for the existence of constant spinor solutions. The mass term enters in the same way for arbitrary dimensions, but for each additional dimension we get an extra spin-connection component, as illustrated in (3.48), which 
enters into the Dirac equation, after multiplying by the curved space gamma matrices, simply as a constant. Therefore as we move onto higher dimensions the constraint on $z$ get recurrently shifted by -1 , starting from the value when $d=2+1^{7}$. The same chiral modes that are obtained in the previous section appear also here. However, in dimensions higher than $d=2+1$, spinor dimensions increase such that the constraints from Maxwell's equations become a much weaker restriction and it should then be possible generally to switch on momentum in other directions. As a result our solution would not be able to capture the back-reaction of a general state where modes with non-trivial momenta $k$ are occupied.

\subsection{Can there be an exact Lifshitz solution?}

Given our analysis with the Chern-Simons theory and the form of the probe fermion stress tensor, it would be interesting to look for exact Lifshitz solutions. However, in the $k_{\mu}=0$ case in $2+1$ dimensions we can show that this is not possible. Note that in light of our discussion of the semi-classical analysis, this will only make sense if the charge $q$ is large compared to the mass. The following should only be treated schematically and we leave a more rigorous analysis for future work.

We will again start with the Einstein-Maxwell-Dirac system in $2+1$ dimensions and look for an exact solution to the equations of motion in the background given in eq.(2.6). The set of equations are

$$
\begin{array}{r}
\Gamma^{\mu} D_{\mu} \psi-m \psi=0, \quad \nabla^{\nu} F_{\mu \nu}=j_{\mu}, \\
R_{\alpha \beta}-\frac{1}{2} g_{\alpha \beta} R-\frac{d(d-1)}{2 L^{2}} g_{\alpha \beta}-\frac{1}{2}\left(F_{\alpha \gamma} F_{\beta}^{\gamma}-\frac{1}{4} F^{2} g_{\alpha \beta}\right)=\frac{1}{2} T_{\alpha \beta}^{f} .
\end{array}
$$

Since we are looking for field configurations depending only on $r$, the left hand side of the $A_{r}$ equation of motion vanishes trivially. This imposes a chirality constraint on the components of the Dirac fermion, namely,

$$
\psi_{+}=\psi_{-} .
$$

Inserting this condition back into the Dirac equation, we get two equations for $\psi_{+}$, which are inconsistent unless

$$
A_{x}=-r^{z-1} A_{t} .
$$

Using this condition the Dirac equation can be solved,

$$
\psi_{+}(r)=\sqrt{r} p_{1} r^{\frac{z-2 m}{2}} .
$$

We can then substitute this solution and the relation between $A_{x}$ and $A_{t}$, in the Einstein equation. The $x-r$ component of the Einstein equation then implies that $\psi_{+}$is a real function and as a result the integration constant $p_{1}$ is real. Having done this, we can solve the $A_{t}$ component of the Maxwell equation, which yields

$$
A_{t}(r)=c_{2}+\frac{c_{1} r^{1-z}}{1-z}-\frac{2 p_{1}^{2} q r^{1-2 m}}{(1-2 m)(2 m-z)} .
$$

Finally we can solve for the remaining components of the Einstein equations. Although we have four equations, only two of them are independent. After having already determined fermion and gauge fields, these two equations are purely algebraic in nature and they constrain the remaining undetermined integration constants, $c_{1}, c_{2}$ and $p_{1}$ and the parameter $z$. Unfortunately, there is

\footnotetext{
${ }^{7}$ Note also that in all these solutions $T_{t}^{t}-T_{r}^{r}=0$.
} 
no consistent choice of these constants that can satisfy both equations. The same analysis can be applied generally for $k_{\mu} \neq 0$, which suggests that Lifshitz spacetimes are not exact solution to the equations of motion. This however does not contradict the fact that in the WKB limit it becomes an approximate solution.

\section{Discussion}

Motivated by results of [4], we studied a system of self-gravitating charged Dirac fermions and showed that non-relativistic Schrödinger metrics exist as exact solutions ${ }^{8}$. In $2+1$ dimensions, this was anticipated by studying the three dimensional Einstein-Maxwell-Chern-Simons system. Although the gauge Chern-Simons term does not couple to the metric, it affects the background by modifying the Maxwell equations. We found that in the presence of gauge and/or gravitational Chern-Simons term, one generically finds Schrödinger spacetime as a suitable background.

One may be worried that by treating the problem involving fermions semi-classically, we could be missing important higher order effects. For starters, the effects of quantum gravity are under control as we are implicitly assuming that the gravitational coupling is small. Next, one could be worried that since there are a large number of fermions, there could be an enhancement of higher loop effects by their presence. However, as demonstrated in a general toy-model in Appendix D, as long as the number of modes $N$ excited is large, the tree level contribution from contraction with the external state would be boosted by factors of $N$ relative to quantum loops at each order of perturbation in the boson-fermion interaction. This gives support to our semi-classical treatment.

Since in the flat space, (charged) fermions induce gauge and gravitational Chern-Simons terms through parity anomaly ${ }^{9}$, the result obtained in the Einstein-Maxwell-Chern-Simons was a precursor to our expectation of back-reaction of fermions on AdS space. Armed with this result we studied fermions in the Schrödinger background in Einstein-Maxwell theory without ChernSimons terms, anticipating that the Schrödinger spacetime is the fully back-reacted geometry of the fermions in AdS space. We indeed found a consistent solution to the gravity-Maxwellfermion system in the Schrödinger spacetime in arbitrary dimension for the critical exponent $z= \pm 2 m L-(D-2)$, where $D$ is the number of bulk spacetime dimensions.

The emergence of Schrödinger spacetime can also be seen perturbatively. Let us start with $\mathrm{AdS}_{3}$ spacetime with metric

$$
d s^{2}=L^{2}\left(-4 r d t d x+\frac{d r^{2}}{4 r^{2}}\right)
$$

and consider gauge field components $A_{\mu}$ taking at most constant values. We can then solve the Dirac equation for a fermion with charge $q$ and mass $m$ in this background. The solution is

$$
\psi(r, t, x)=r^{\frac{m}{2}-\frac{3}{4}} \exp (-i \omega t)\left(\begin{array}{l}
1 \\
1
\end{array}\right) .
$$

We then look at the back-reaction of this solution on the background metric and gauge fields. Since we are treating this fermion solution perturbatively, the back-reaction will be tiny (proportional to $\hbar$ ) but indicative of the effect of macroscopic droplet of these fermion on the background. With this in mind we evaluate fermion current and energy momentum tensor and feed

\footnotetext{
${ }^{8}$ It is important to note that the fermion gas we are considering is a smooth density distribution, which alleviates Coulomb repulsion particularly along the $x$-direction, that otherwise would have made the gas unstable.

${ }^{9}$ Fermion mass term, in fact, breaks parity symmetry explicitly in $2+1$ dimensions. On the other hand in $3+1$ dimensions it does not break parity.
} 
it back into equations of motion of the background. Solution to equations with back-reaction of fermions generate non-trivial gauge field component $A_{t}$ and metric component $g_{t t}$, which take the form

$$
\begin{aligned}
A_{t}(r) & =\hbar\left(c_{2}+c_{1} \ln r+\frac{4 q r^{m-1 / 2}}{(2 m-1)^{2}}\right) \\
g_{t t} & =\hbar \frac{1}{(2 m-1)^{2}}\left(\frac{-4 q^{2} r^{2 m-1}}{\left(2 m^{2}-3 m+1\right)}+e_{1} \ln r+e_{2} r^{m-1 / 2} \ln r\right),
\end{aligned}
$$

where $c_{i}$ are constants of integration and the $e_{i}$ 's are fixed in terms of $c_{i}$ via Einstein equation. In particular, setting $c_{1}=0$ sets both $e_{i}=0$. In this limit we are actually reduced to the original solution we found previously in (3.10), (3.32) and (3.37). The fermions only appear differently because we have picked a different set of vierbeins to suit our purpose here starting off from a off-diagonal metric.

It is interesting to note that for $z>0$ the Schrödinger backgrounds are horizon-free and as a result do not have macroscopic entropy associated with them. The phenomenon of disappearance of the horizon due to fermion back-reaction is similar to what was proposed in [4]. It would be interesting to find a dual description of our solutions. In the $2+1$ dimensions we studied the back-reacted geometry supported either by the Chern-Simons terms or by fermions. As mentioned earlier, around flat backgrounds it is known that Chern-Simons terms are induced by one loop effects in the fermionic theory $[8,7]$. In the context of curved space we have two Chern-Simons terms, gauge and gravitational. The Schrödinger metric obtained in the ChernSimons theory has (up to sign) two new exponents apart from the standard one corresponding to AdS space; $z=\mp 4 \mu_{F}$ and $z=\left(\mu_{G} \mp 1\right) /\left(2 \mu_{G}\right)$ (see (2.5)). On the other hand, in the fermionic theory we have only one exponent (up to a sign of the fermion mass term). It is tempting to conjecture that even in curved spacetimes, the fermions would induce Chern-Simons terms. It would be interesting to directly relate these two theories and compare their resulting exponent $z$.

Let us make a brief comment on the Maxwell-Chern-Simons theory. Around flat spacetime it is possible to arrange the coefficient of gauge Chern-Simons term and the number of fermion species to switch off the gravitational Chern-Simons term[9]. It turns out that this system has already been studied in [21,22] and [23]. In [23], it was proved that there exist no black hole solutions in this theory. The existence of Chern-Simons term in $2+1$ dimensions implies the presence of a chiral anomaly in the $1+1$ dimensional theory [24]. The induced chiral anomaly in the boundary theory is canceled by the chiral current in the boundary theory. In [22], it was found (after transforming to our conventions), that regular black hole solutions only exist if $\mu_{F} \mu_{G}>1 / 6$. This is reminiscent of the condition (C.11).

It has been shown in [4] that Lifshitz metrics are solutions when a WKB approximation is made. We leave it as an open problem to examine if they are also exact solutions. In light of our analysis using the Chern-Simons theory and the self-gravitating fermions, this is unlikely to happen in $2+1$ dimensions. However in higher dimensions, there are more ways to solve the constraint $j_{r}=0$ than in $2+1$ dimensions. So it could very well be that both Lifshitz and Schrödinger metrics exist as exact solutions. It will be exciting to find new backgrounds from self-gravitating fermions. Of course in general, this is a very difficult problem.

\section{Acknowledgments}

We would like to thank Sumit Das, Suresh Govindarajan, T. Padmanabhan, Sumathi Rao, Harvey Reall, Ashoke Sen, Yanwen Shang, Brian Shieh and Subir Sachdev for discussions. 
We also thank Sean Hartnoll and David Tong for numerous email exchanges which helped us understand what we are doing better. We would particularly like to thank Rob Myers for detailed discussions and for several excellent suggestions. We have made some use of the free Mathematica package, tools of tensor calculus, in our computations. Research at Perimeter Institute is supported by the Government of Canada through Industry Canada and by the Province of Ontario through the Ministry of Research \& Innovation.

\section{A Conventions}

We use the following conventions for three and four dimensions. The three dimensional action with Chern-Simon terms is

$$
I_{3}=\frac{1}{2 \ell_{p}} \int d^{3} x\left[\sqrt{-g}\left(R+\frac{2}{L^{2}}-\frac{1}{4} F_{\mu \nu} F^{\mu \nu}\right)+L_{M C S}+L_{G C S}\right]
$$

where the Maxwell Chern Simons and gravitational Chern Simons are given by

$$
\begin{aligned}
L_{M C S} & =\mu_{F} \epsilon^{\alpha \beta \gamma} A_{\alpha} F_{\beta \gamma}, \\
L_{G C S} & =\mu_{G} \epsilon^{\lambda \mu \nu} \Gamma_{\lambda \beta}^{\alpha}\left(\partial_{\mu} \Gamma_{\alpha \nu}^{\beta}+\frac{2}{3} \Gamma_{\mu \gamma}^{\beta} \Gamma_{\nu \alpha}^{\gamma}\right) .
\end{aligned}
$$

The four dimensional action is

$$
I_{4}=\frac{1}{2 \ell_{p}^{2}} \int d^{4} x\left[\sqrt{-g}\left(R+\frac{6}{L^{2}}-\frac{1}{4} F_{\mu \nu} F^{\mu \nu}\right)\right] .
$$

The Einstein equation is

$$
R_{\mu \nu}-\frac{1}{2} g_{\mu \nu} R+\Lambda g_{\mu \nu}=\frac{1}{2} T_{\mu \nu}
$$

where $\Lambda$ is the cosmological constant in appropriate dimensions and $T_{\mu \nu}$ is the matter stress energy tensor. The matter stress energy tensor with this choice of conventions is defined as

$$
T_{\mu \nu}=-\frac{2}{\sqrt{-g}} \frac{\delta S}{\delta g^{\mu \nu}} .
$$

The generic asymptotically AdS metric is written as

$$
d s^{2}=-\frac{r^{2}}{L^{2}} f(r) d t^{2}+\frac{L^{2}}{r^{2}} \frac{d r^{2}}{f(r)}+\frac{r^{2}}{L^{2}} d x_{i}^{2} .
$$

In absence of Chern-Simons terms in three dimensions it can be shown that (A.8) solves equations of motion to give charged BTZ black hole solution[25, 26, 27] with

$$
f(r)=1-\frac{q^{2} L^{2}}{4 r^{2}}-\frac{q^{2} L^{2}}{2 r^{2}} \ln \frac{r}{r_{*}}, \quad A_{t}=q \ln \frac{r}{r_{0}},
$$

where $r_{0}$ is the location of outer horizon. The condition for existence of two horizons is $q^{2} L^{2} \geq$ $4 r_{*}^{2}$ and the extremality condition is $r_{*}=q L / 2$.

In four dimensions, (A.8) solves the equations of motion to give charged Reissner-Nordström black hole solution, with behaviour of $f(r)$ and $A_{t}(r)$ given by

$$
f(r)=1-\frac{M}{r^{3}}+\frac{q^{2} L^{2}}{4 r^{4}}, \quad A_{t}(r)=q\left(\frac{1}{r}-\frac{1}{r_{0}}\right) .
$$


In order to have double horizon $27 M^{4} \geq 4 q^{6} L^{6}$ and the equality corresponds to extremal solution.

The near horizon metric in the extremal limit is given by $\mathrm{AdS}_{2} \times M_{n}$, where $M_{n}$ is an $n$ dimensional manifold, which in our case is either $R^{n}$ or $S^{n}$. Our convention for $\mathrm{AdS}_{2}$ metric in Poincare coordinates is

$$
d s_{A d S 2}^{2}=-\frac{r^{2}}{\tilde{L}^{2}} d t^{2}+\frac{\tilde{L}^{2}}{r^{2}} d r^{2}
$$

where $\tilde{L}$ is the radius of $\mathrm{AdS}_{2}$. In $2+1$ dimensions, according to our conventions,

$$
A_{t}=\sqrt{2} \frac{r}{\tilde{L}}, \quad \tilde{L}=\frac{L}{\sqrt{2}},
$$

while in $3+1$ dimensions,

$$
A_{t}=\sqrt{2} \frac{r}{\tilde{L}}, \quad \tilde{L}=\frac{L}{\sqrt{6}}
$$

\section{B Spin connection and the Dirac Equation}

Consider a general metric of the form

$$
d s^{2}=-g_{t t}(r) d t^{2}+g_{r r}(r) d r^{2}+g_{x x}(r) d x^{2}+g_{i i}(r) d x^{i} d x^{i}+2 g_{t x}(r) d t d x,
$$

with all metric components having only radial dependence. We will use $\mu, \nu, \cdots$ to denote curved spacetime indices and $i, j, \cdots$ to denote curved spatial indices. We will reserve index ' $d$ ' for denoting flat coordinate related to curved index $r$ by vielbein. Flat space indices for boundary coordinates are denoted by $a, b, \cdots$.

In this case, following choice of vielbeins is suitable for both diagonal metrics as well as Schrödinger metrics

$$
\begin{aligned}
& e^{0}=\sqrt{g_{t t}} d t-\frac{g_{t x}}{\sqrt{g_{t t}}} d x, e^{d}=\sqrt{g_{r r}} d r \\
& e^{1}=\frac{\sqrt{g_{t t} g_{x x}+g_{t x}^{2}}}{\sqrt{g_{t t}}} d x, \quad e^{a}=\sqrt{g_{i i}} d x^{i}, a=2,3, \cdots .
\end{aligned}
$$

It is straightforward to derive components of spin connections from them and they are given by

$$
\begin{aligned}
& \omega_{d 0}=\frac{1}{2} \frac{g_{t t}^{\prime}(r)}{\sqrt{g_{t t} g_{r r}}} d t-\frac{1}{2} \frac{g_{t x}}{\sqrt{g_{t t} g_{r r}}} \frac{g_{t t}^{\prime}(r)}{g_{t t}} d x, \\
& \omega_{d 1}=-\frac{1}{2 \sqrt{(-\operatorname{det} g) g_{t t}}}\left[\left(g_{t t} g_{x x}^{\prime}(r)+g_{t x} g_{t x}^{\prime}(r)\right) d x+\left(g_{t t} g_{t x}^{\prime}(r)-g_{t x} g_{t t}^{\prime}(r)\right) d t\right], \\
& \omega_{d i}=\frac{g_{i i}^{\prime}(r)}{2 \sqrt{g_{r r} g_{i i}}} d x^{i},
\end{aligned}
$$

where primes one the metric components are derivatives with respect to their argument.

Let us now look at the Dirac equation in different backgrounds. The Dirac equation for a particle with mass $m$ and charge $e$ takes the form

$$
(\not D-m) \psi=0
$$

where

$$
\not D=\Gamma^{c} e_{c}^{\mu}\left(\partial_{\mu}+\frac{1}{4} \omega_{\mu a b} \Gamma^{a} \Gamma^{b}+i q A_{\mu}\right) .
$$


To solve the Dirac equation we will use the following $\Gamma$-matrix convention for $2+1$ dimensions,

$$
\Gamma^{1}=\sigma_{3}, \quad \Gamma^{0}=i \sigma_{2}, \quad \Gamma^{d}=\sigma_{1} .
$$

Our $3+1$ dimensional $\Gamma$-matrix convention is

$$
\Gamma^{d}=\left(\begin{array}{cc}
I & 0 \\
0 & -I
\end{array}\right), \quad \Gamma^{a}=\left(\begin{array}{cc}
0 & \sigma^{a} \\
\sigma^{a} & 0
\end{array}\right), \quad a=0,1,2
$$

Where $I$ is $2 \times 2$ identity matrix and $\sigma^{a}$ are Pauli matrices.

\section{Stress tensor of fermionic probes in AdS}

We will be considering fermions in curved space. We shall begin presenting ${ }^{10}$ explicitly the fermionic contribution to the stress tensor [19]. The quadratic action for a charged massive Dirac spinor is

$$
S_{\text {Dirac }}=\frac{1}{2 \ell_{p}^{d-1}} \int d^{d+1} x \sqrt{-g} i\left(\bar{\psi} \Gamma^{a} e_{a}^{\mu} D_{\mu} \psi-m \bar{\psi} \psi\right)
$$

where

$$
\begin{aligned}
& D_{\mu} \psi=\left(\partial_{\mu}+\frac{i}{2} \omega_{\mu}^{a b} \Gamma_{a b}+i q A_{\mu}\right) \psi, \quad \bar{\psi} \overleftarrow{D}_{\mu}=\left(\partial_{\mu} \bar{\psi}-\frac{i}{2} \omega_{\mu}^{a b} \bar{\psi} \Gamma_{a b}-i q A_{\mu} \bar{\psi}\right) \\
& \bar{\psi}=\psi^{\dagger} \Gamma^{0}, \quad \Gamma_{a b}=-\frac{i}{4}\left[\Gamma_{a}, \Gamma_{b}\right]
\end{aligned}
$$

The corresponding stress tensor is

$$
T_{\mu \nu}^{f}=\frac{1}{2}\left(-i \bar{\psi} \Gamma_{(\mu} D_{\nu)} \psi+i \bar{\psi} \overleftarrow{D}_{(\mu} \Gamma_{\nu)} \psi\right)
$$

and symmetrization is denoted by $V_{(\mu}^{1} V_{\nu)}^{2}=\frac{1}{2}\left(V_{\mu}^{1} V_{\nu}^{2}+V_{\nu}^{1} V_{\mu}^{2}\right)$.

\section{C.1 $\quad \operatorname{AdS}_{2} \times R$}

The background is, following our conventions,

$$
d s_{A d S 2 \times R}^{2}=-\frac{r^{2}}{\tilde{L}^{2}} d t^{2}+\frac{\tilde{L}^{2}}{r^{2}} d r^{2}+d x^{2}, \quad A_{t}=\frac{\sqrt{2} r}{\tilde{L}},
$$

and the only non-vanishing spin connection is,

$$
\omega_{10}=\frac{r}{\tilde{L}^{2}} d t
$$

Taking a Dirac spinor of the form

$$
\psi=\left(\begin{array}{c}
\psi_{+} \\
\psi_{-}
\end{array}\right)
$$

and assuming only $r$ dependence, the Dirac equation is given explicitly by

$$
2 r \psi_{ \pm}^{\prime}+2 i q \sqrt{2} \psi_{ \pm}+(1 \mp 2 m \tilde{L}) \psi_{\mp}=0 .
$$

\footnotetext{
${ }^{10}$ Note that [19] use the mostly negative convention while we follow the more standard GR convention of mostly positive.
} 
The solution is simply

$$
\psi^{ \pm}=r^{-\frac{1}{2} \pm \delta}\left(\begin{array}{c}
p_{1}^{ \pm} \\
p_{2}^{ \pm}
\end{array}\right), \quad p_{2}^{ \pm}= \pm \frac{i p_{1}^{ \pm}(\delta \mp m \tilde{L})}{\sqrt{2} L q}, \quad \delta=\tilde{L} \sqrt{m^{2}-2 q^{2}},
$$

for some constants $p_{+}^{ \pm}$. Switching on one of the two solutions leads to

$$
\begin{aligned}
T_{t t}^{f} & =\frac{\left|p_{1}^{ \pm}\right|^{2} \sqrt{2} m(m \tilde{L} \mp \delta)}{\tilde{L}^{3} q} r^{1 \pm 2 \delta}, \\
T_{x t}^{f} & = \pm\left|p_{1}^{ \pm}\right|^{2} \frac{m \tilde{L}(2 \delta \mp 1)+\delta \mp 2 \delta^{2}}{2 \sqrt{2} q \tilde{L}^{3}} .
\end{aligned}
$$

Note the appearance of $T_{x t}$ components. The $r r$ component contributes only from cross terms when both solutions are switched on. Note that the Ricci tensor for the background metric is simply

$$
R_{t t}=\frac{r^{2}}{\tilde{L}^{4}}, \quad R_{r r}=-\frac{1}{r^{2}} .
$$

Therefore, in the near horizon limit $r \rightarrow 0$, the solution scaling with $r^{-\left(\frac{1}{2}+\delta\right)}$ would eventually become dominant over the background such that the probe approximation breaks down. For the other solution that scales as $r^{-\left(\frac{1}{2}-\delta\right)}$ however, the probe approximation is good all the way to the horizon if $\delta>1$, which gives

$$
m^{2}>2 q^{2}+1
$$

When the mass is smaller than $2 q^{2}+1$, back reaction in the near horizon region cannot be ignored, with the likely result of destroying the horizon altogether. Therefore in the large mass and charge limit we recover the assertion in [4] that roughly horizon is destroyed whenever $e q>$ $m$, where $e=\sqrt{2}$ is the background electric field. Note also that these solutions independently satisfy

$$
T_{t}^{t}-T_{r}^{r}=-\frac{\left|p_{1}^{ \pm}\right|^{2} \sqrt{2} m(m \tilde{L} \mp \delta)}{\tilde{L} q} r^{-1 \pm 2 \delta}<0
$$

\section{C.2 $\quad \mathrm{AdS}_{2} \times R^{2}$}

Let us repeat the exercise in the previous section in $\mathrm{AdS}_{2} \times R^{2}$. A static solution of a 4component Dirac fermion would be given by

$$
\psi=\psi_{+} r^{-\frac{1}{2}+\delta}+\psi_{-} r^{-\frac{1}{2}-\delta}, \quad \delta=\tilde{L} \sqrt{m^{2}-2 q^{2}},
$$

where

$$
\psi_{ \pm}=\left(\begin{array}{c}
\psi_{ \pm}^{1} \\
\psi_{ \pm}^{2}
\end{array}\right), \quad \psi_{ \pm}^{2}= \pm \frac{\left(\frac{\delta}{\tilde{L}} \mp m\right)}{\sqrt{2} q \tilde{L}}\left(\sigma_{1} \psi_{ \pm}^{1}\right),
$$

where $\psi_{ \pm}^{i}$ are two component constant spinors and $\sigma_{i}$ are the Pauli matrices. The non-vanishing contribution of $\psi_{+}$to the energy-momentum tensor is given by

$$
\begin{aligned}
T_{t t}^{f} & =\psi_{ \pm}^{1 \dagger} \psi_{ \pm}^{1} \frac{r^{1 \pm 2 \delta}\left(\tilde{L}^{2}\left(m^{2}+2 q^{2}\right) \mp 2 \tilde{L} m \delta+\delta^{2}\right)}{\sqrt{2} \tilde{L}^{4} q} \\
T_{t x}^{f} & =\psi_{ \pm}^{1 \dagger} \sigma_{3} \psi_{ \pm}^{1} \frac{r^{ \pm 2 \delta}\left(\tilde{L}^{2}\left(m^{2}-2 q^{2}\right)+(\delta \mp 1) \delta+\tilde{L}(m \mp 2 m \delta)\right)}{2 \sqrt{2} \tilde{L}^{3} q} \\
T_{t y}^{f} & =-\psi_{ \pm}^{1 \dagger} \sigma_{2} \psi_{ \pm}^{1} \frac{r^{ \pm 2 \delta}\left(\tilde{L}^{2}\left(m^{2}-2 q^{2}\right)+(\delta \mp 1) \delta+\tilde{L}(m \mp 2 m \delta)\right)}{2 \sqrt{2} \tilde{L}^{3} q}
\end{aligned}
$$


As in the previous section, the energy-momentum clearly illustrates again that back-reaction is severe in the near horizon region generally for $\sqrt{2} q>m$ Note that here

$$
T_{t}^{t}-T_{r}^{r}=-\psi_{ \pm}^{1 \dagger} \psi_{ \pm}^{1} \frac{r^{-1 \pm 2 \delta}\left(\tilde{L}^{2}\left(m^{2}+2 q^{2}\right) \mp 2 \tilde{L} m \delta+\delta^{2}\right)}{\sqrt{2} \tilde{L}^{2} q}<0
$$

\section{C.3 $\quad \mathrm{AdS}_{2} \times S^{2}$}

An entirely parallel story goes through if one were to replace $R^{2}$ in the previous sub-section by $S^{2}$. Namely

$$
\psi=\psi_{+} r^{-\frac{1}{2}+\delta}+\psi_{-} r^{-\frac{1}{2}-\delta}, \quad \delta=\tilde{L} \sqrt{m^{2}-2 q^{2}},
$$

where, however even the lowest mode contains non-trivial angular dependence,

$$
\psi_{ \pm}=\frac{1}{\sqrt{\sin \theta}}\left(\begin{array}{c}
\psi_{ \pm}^{1} \\
\psi_{ \pm}^{2}
\end{array}\right), \quad \psi_{ \pm}^{2}= \pm \frac{\left(\frac{\delta}{\tilde{L}} \mp m\right)}{\sqrt{2} q \tilde{L}}\left(\sigma_{1} \psi_{ \pm}^{1}\right),
$$

The energy-momentum tensor here is similarly,

$$
\begin{aligned}
T_{t t}^{f} & =\csc \theta \psi_{ \pm}^{1 \dagger} \psi_{ \pm}^{1} \frac{r^{1 \pm 2 \delta}\left(\tilde{L}^{2}\left(m^{2}+2 q^{2}\right) \mp 2 \tilde{L} m \delta+\delta^{2}\right)}{\sqrt{2} \tilde{L}^{4} q} \\
T_{t \theta}^{f} & =\csc \theta \psi_{ \pm}^{1 \dagger} \sigma_{3} \psi_{ \pm}^{1} \frac{r^{ \pm 2 \delta}\left(\tilde{L}^{2}\left(m^{2}-2 q^{2}\right)+(\delta \mp 1) \delta+\tilde{L}(m \mp 2 m \delta)\right)}{2 \sqrt{2} \tilde{L}^{3} q} \\
T_{t \phi}^{f} & =\frac{-\sqrt{2} \tilde{L} \cos \theta}{8 q r} T_{t t}^{f}-\psi_{ \pm}^{1 \dagger} \sigma_{2} \psi_{ \pm}^{1} \frac{r^{ \pm 2 \delta}\left(\tilde{L}^{2}\left(m^{2}-2 q^{2}\right)+(\delta \mp 1) \delta+\tilde{L}(m \mp 2 m \delta)\right)}{2 \sqrt{2} \tilde{L}^{3} q}
\end{aligned}
$$

The null energy condition is the same as eq. (C.19).

\section{A toy model justification for our semi-classical treatment}

Our discussion is largely based on the assumption that in the presence of a large fermi-gas, one can treat the background classically, while the fermions propagate in this mean-field background quantum-mechanically. We consider here a toy model where fermions are coupled to bosons. In the case where the fermion fields appear only up to quadratic order, we can obtain the 1loop effective action of the bosons after integrating out the fermions in an $N$-fermion state. It turns out, in the large $N$-limit and to leading order in the boson-fermion coupling $g$, the effective equations of motion of the bosons are equivalent to the original one, except the fermion operators are replaced by their expectation values evaluated on the $N$-fermion state. The toy model gives us more confidence in the methods we advocate in this paper. It is also noteworthy that the approach of treating back-reacting fermions on a mean-field background and obtaining self-consistent set of solutions to all the equations of motion with an explicit built-in Fermisurface, is a well-known strategy in nuclear physics[15], which is equivalent in various limits to the Hartree-Fock approximation.

Our discussion here will follow closely [28]. The (Euclidean) action of our toy-model at temperature $T=\beta^{-1}$ in $(0+1)$ dimension is as follows:

$$
S=S_{b}+S_{f}+S_{i n t}, \quad S_{b}=\frac{1}{2} \int_{0}^{\beta} d \tau \dot{\phi}^{2}+m_{b}^{2} \phi^{2},
$$




$$
S_{f}+S_{i n t}=\int_{0}^{\beta} d \tau \sum_{i} b_{i}^{\dagger} \dot{b}_{i}+\left(m_{i}-\mu_{i}\right) b_{i}^{\dagger} b_{i}-g \phi b_{i}^{\dagger} b_{i}
$$

where we have introduced many species of fermionic fields $b_{i}$ and a corresponding chemical potential $\mu_{i}$ for each species, which are to be determined from some given occupation of the fermionic states. The partition function is given by

$$
Z=\int D[\phi] \prod_{i} D\left[i b_{i}^{\dagger}\right] D\left[b_{i}\right] \exp \left(\frac{-S}{\hbar}\right)
$$

Since the action is quadratic in the fermions, the path-integral can be readily done. Expressing the fields as

$$
b_{i}=\sum_{n} e^{-i \omega_{n}^{f} \tau} b_{i ; n}, \quad \phi=\sum_{n} e^{-i \omega_{n}^{b} \tau} \phi_{n}
$$

The $\omega_{n}$ here are thus Matsubara frequencies given by

$$
\omega_{n}^{f}=(2 n+1) \pi T, \quad \omega_{n}^{b}=2 n \pi T,
$$

and $S_{f}+S_{\text {int }}$ can be re-written as

$$
S_{f}+S_{i n t}=\sum_{i, m, n} i b_{i ; m}^{\dagger} \mathcal{D}_{m n}^{i} b_{i ; n}, \quad \mathcal{D}_{m n}^{i}=-i \beta\left(\left(-i \omega_{n}+\mu_{i}-m_{i}\right) \delta_{m n}-g \phi_{m-n}\right) .
$$

Integrating out the fermions one is then left with

$Z=\int D[\phi] \exp \left(-\frac{S_{b}}{\hbar}\right) \operatorname{det}\left(\frac{\mathcal{D}}{\hbar}\right)=\operatorname{det}\left(\beta\left(-i \omega_{n}+\mu_{i}-m_{i}\right)\right) \int D[\phi] \exp \left(-\frac{S_{b}}{\hbar}\right) \operatorname{det}\left(1-\frac{g \phi_{m-n}}{-i \omega_{n}+\mu_{i}-m_{i}}\right)$.

To lowest order in the coupling $g$, the fermion contribution to the free energy evaluates to [28], using various summation formula,

$$
\begin{aligned}
\frac{F_{f}}{T} \quad & =-\ln Z_{f}=-\sum_{n, i} \ln \left[\beta\left(-i \omega_{n}+\mu_{i}-m_{i}\right)\right] \\
& =-\frac{1}{2} \sum_{i}\left[\beta^{2}\left(\sum_{n}\left(\omega_{n}^{2}+\left(\mu_{i}-m_{i}\right)^{2}\right)\right)\right] \\
& =-\sum_{i} \ln \left(1+e^{-\beta\left(m_{i}-\mu\right)}\right),
\end{aligned}
$$

where we have dropped the contribution of vacuum energy which has to be regulated. We can determine the chemical potentials by

$$
\partial_{\mu_{i}}(T \ln Z) \sim \frac{1}{e^{\beta\left(m_{i}-\mu_{i}\right)}+1}=N_{i} .
$$

which is precisely the Fermi-distribution. The ground state

$$
|Q\rangle=\prod_{i} b_{i}^{\dagger}|0\rangle,
$$

corresponding to a system of $N$ fermions with fermi-energy $E_{F}$ can thus be obtained by picking

$$
\mu_{i}=E_{F} .
$$


In which case the Fermi-distribution behaves like a step function, giving occupation $N_{i}=1$ for all single particle states below $E_{f}$. Now returning to the full path integral, we have

$$
\begin{aligned}
Z & =\int D[\phi] \exp \left(-\frac{S_{b}}{\hbar}\right) \exp \left(\ln \operatorname{det}\left(\frac{D}{\hbar}\right)\right) \\
& \sim \int D[\phi] \exp \left(-\frac{S_{b}}{\hbar}\right) \exp \left(\sum_{i} \beta m_{i}+\ln \left(1+e^{-\beta\left(m_{i}-E_{f}-g \phi_{0}\right)}\right)-\ln \hbar\right),
\end{aligned}
$$

where in the second line we have taken the leading $g$ approximation, so that in the determinant of a matrix of the form

$$
\left(\begin{array}{ccc}
\left(1+g \phi_{0}\right) & g \phi_{1} & \cdots \\
g \phi_{-1} & \left(1+g \phi_{0}\right) & \ldots \\
\vdots & \vdots & \ddots
\end{array}\right)
$$

the leading $g$ dependence came from the trace, which include only the diagonal components with $\phi_{(m-m=0)}$. The off-diagonal components can only arise in higher $g$ corrections.

Since in the zero-temperature limit $\beta \rightarrow \infty$ the factor $e^{-\beta\left(m_{i}-E_{f}\right)}$ approaches infinity for $m_{i}<E_{f}$ and zero otherwise, we have

$$
\begin{aligned}
Z & \sim \int D[\phi] \exp \left(-\frac{S_{b}}{\hbar}\right) \exp \left(\sum_{i=\text { filled states }}-\beta\left(m_{i}-E_{f}-g \phi_{0}\right)\right) \\
& \sim \int D[\phi] \exp \left(-\frac{S_{b}}{\hbar}\right) \exp \left(-\beta\left(N g \phi_{0}\right)+\ldots\right),
\end{aligned}
$$

where the vacuum energy that is supposed to be regulated, and other pieces independent of $\phi$ are denoted in .... Note that to leading order in $g$ the effective action of the bosons after integrating out the fermions, is precisely as if we replace the fermion operators by their expectation values in the $N$-fermion state. For back-reaction on the bosons to be important however, we need

$$
N g \sim \frac{1}{\hbar}
$$

Note also that the approximation we are taking in the partition function is equivalent to, from the perspective of perturbation theory via Feynman diagrams, only the tree diagrams i.e. The perturbation operators are only contracting with external states. Consider for example the leading order $g$ correction to any expectation values of some arbitrary operators $\mathcal{O}$ in the presence of the interaction terms,

$$
\left\langle Q\left|\mathcal{O} \int \phi \sum_{i} b_{i}^{\dagger} b_{i}\right| Q\right\rangle
$$

we see that contraction of the $b_{i}$ 's with the external creation operators in $|Q\rangle$ result in a factor of $N$, whereas contraction among the $b_{i}$ 's, corresponding to loop diagrams, is only of order 1 relatively. Similarly at order $g^{2}$, we have

$$
\frac{1}{2 !} \iint \sum_{i, j} \phi \phi\left\langle Q\left|\mathcal{O}\left(b_{i}^{\dagger} b_{i}\right)\left(b_{j}^{\dagger} b_{j}\right)\right| Q\right\rangle,
$$

where contraction of the $b$ 's with the external state would again give rise to an extra factor of roughly $N(N-1) \sim N^{2}$ relative to contraction among the $b$ and $b^{\dagger} .{ }^{11}$ In the limit where $N$ is large and $g$ is small, the loop diagrams would be suppressed relative to tree diagrams.

\footnotetext{
${ }^{11}$ Note that the combinatoric factor of 2 ! would appear in either internal contractions or contraction with the external state. We have also made used of the fact that states with different excitations are orthogonal to each other. Therefore having chosen a particular contraction for the $b_{j}$ 's on $|Q\rangle$, the contraction of $b_{i}^{\dagger}$ with $\langle Q|$ is completely determined. This explains why there is only a factor of $N(N-1)$, rather than the square of it.
} 
To summarize, in the limit where $N g \sim \hbar^{-1}$, the back-reaction of the fermi-gas on the bosons become significant, but at the same time we have the quantum loops under control because $g$ is small. Particularly, the leading $g$ effect on the bosons can be captured by replacing all the fermionic operators by their expectation values evaluated on the filled state $|Q\rangle$ in the equations of motion of the bosons. In fact the coupling to the bosonic field $g \phi b^{\dagger} b$ could have been generalized to some arbitrary one of the form $g V(\phi) b^{\dagger} b$ without altering the conclusion, as long as $g$ is small. The toy model appears to justify the approach taken in this paper in including fermionic back-reactions on a bosonic background.

\section{References}

[1] D. T. Son and A. O. Starinets, "Viscosity, Black Holes, and Quantum Field Theory," Ann. Rev. Nucl. Part. Sci. 57, 95 (2007) [arXiv:0704.0240 [hep-th]].

T. Schafer and D. Teaney, "Nearly Perfect Fluidity: From Cold Atomic Gases to Hot Quark Gluon Plasmas," Rept. Prog. Phys. 72, 126001 (2009) [arXiv:0904.3107 [hep-ph]].

A. Sinha and R. C. Myers, "The viscosity bound in string theory," Nucl. Phys. A 830, 295C (2009) [arXiv:0907.4798 [hep-th]].

[2] S. A. Hartnoll, "Lectures on holographic methods for condensed matter physics," Class. Quant. Grav. 26, 224002 (2009) [arXiv:0903.3246 [hep-th]].

C. P. Herzog, "Lectures on Holographic Superfluidity and Superconductivity," J. Phys. A 42, 343001 (2009) [arXiv:0904.1975 [hep-th]].

J. McGreevy, "Holographic duality with a view toward many-body physics," arXiv:0909.0518 [hep-th].

G. T. Horowitz, "Introduction to Holographic Superconductors," arXiv:1002.1722 [hep-th].

[3] H. Liu, J. McGreevy and D. Vegh, "Non-Fermi liquids from holography," arXiv:0903.2477 [hep-th].

T. Faulkner, H. Liu, J. McGreevy and D. Vegh, "Emergent quantum criticality, Fermi surfaces, and AdS2," arXiv:0907.2694 [hep-th].

N. Iqbal and H. Liu, "Real-time response in AdS/CFT with application to spinors," Fortsch. Phys. 57, 367 (2009) [arXiv:0903.2596 [hep-th]].

[4] S. A. Hartnoll, J. Polchinski, E. Silverstein and D. Tong, "Towards strange metallic holography," JHEP 1004, 120 (2010) [arXiv:0912.1061 [hep-th]].

[5] Shamit Kachru, Xiao Liu, Michael Mulligan "Gravity Duals of Lifshitz-like Fixed Points," [arXiv:0808.1725 [hep-th]].

[6] L. Y. Hung and A. Sinha, "Holographic quantum liquids in 1+1 dimensions," JHEP 1001, 114 (2010) [arXiv:0909.3526 [hep-th]].

D. Maity, S. Sarkar, N. Sircar, B. Sathiapalan and R. Shankar, "Properties of CFTs dual to Charged BTZ black-hole," [ arXiv:0909.4051 [hep-th]].

[7] D. Boyanovsky and R. Blankenbecler, "Axial and parity anomalies and vacuum charge: A direct approach", Phys. Rev. D 313234 (1985).

[8] A. N. Redlich, "Gauge Noninvariance and Parity Nonconservation of Three Dimensional Fermions", Phys. Rev. Lett. 5218 (1984).

[9] J. J. van der Bij, R. Pisarski, S. Rao, "Topological Mass term for Gravity induced by Matter", Phys. Lett. B 17987 (1986).

[10] S. Deser, R. Jackiw and S. Templeton, "Topologically massive gauge theories," Annals Phys. 140, 372 (1982). 
W. Li, W. Song and A. Strominger, "Chiral Gravity in Three Dimensions," JHEP 0804, 082 (2008) [arXiv:0801.4566 [hep-th]].

[11] A.P. Balachandran, H. Gomm and R.D. Sorkin, "Quantum Symmetries from Quantum Phases, Fermions from Bosons, a $\mathcal{Z}_{2}$ Anomaly, and Galilean Invariance", Nuc. Phys.B281573-6121987.

C. Duval and P. A. Horváthy and L. Palla, "Conformal symmetry of the coupled ChernSimons and gauged non-linear Schrödinger equations." Phys. Lett. B325, 39 (1994) [hepth/9401065].

C. Duval and P. A. Horváthy and L. Palla, "Conformal properties of Chern-Simons vortices in external fields." Phys. Rev. D50, 6658 (1994) [hep-ph/9405229].

K. Balasubramanian and J. McGreevy, "Gravity duals for non-relativistic CFTs," Phys. Rev. Lett. 101, 061601 (2008) [arXiv:0804.4053 [hep-th]].

D. T. Son, "Toward an AdS/cold atoms correspondence: a geometric realization of the Schroedinger symmetry," Phys. Rev. D 78, 046003 (2008) [arXiv:0804.3972 [hep-th]].

A. Adams, K. Balasubramanian and J. McGreevy, "Hot Spacetimes for Cold Atoms," JHEP 0811, 059 (2008) [arXiv:0807.1111 [hep-th]].

C. P. Herzog, M. Rangamani and S. F. Ross, "Heating up Galilean holography," JHEP 0811, 080 (2008) [arXiv:0807.1099 [hep-th]].

J. Maldacena, D. Martelli and Y. Tachikawa, "Comments on string theory backgrounds with non-relativistic conformal symmetry," JHEP 0810, 072 (2008) [arXiv:0807.1100 [hepth]].

E. Imeroni and A. Sinha, "Non-relativistic metrics with extremal limits," JHEP 0909, 096 (2009) [arXiv:0907.1892 [hep-th]].

A. Adams, C. M. Brown, O. DeWolfe and C. Rosen, "Charged Schrodinger Black Holes," Phys. Rev. D 80, 125018 (2009) [arXiv:0907.1920 [hep-th]].

D. Orlando and L. I. Uruchurtu, "Warped anti-de Sitter spaces from brane intersections in type II string theory," JHEP 1006, 049 (2010) [arXiv:1003.0712 [hep-th]].

D. Israel, C. Kounnas, D. Orlando and P. M. Petropoulos, "Electric / magnetic deformations of $\mathrm{S}^{* *} 3$ and $\mathrm{AdS}(3)$, and geometric cosets," Fortsch. Phys. 53, 73 (2005) [arXiv:hepth/0405213].

S. Detournay, D. Orlando, P. M. Petropoulos and P. Spindel, "Three-dimensional black holes from deformed anti de Sitter," JHEP 0507, 072 (2005) [arXiv:hep-th/0504231].

[12] D. Anninos, W. Li, M. Padi, W. Song and A. Strominger, "Warped AdS 3 Black Holes," JHEP 0903, 130 (2009) [arXiv:0807.3040 [hep-th]].

[13] D. R. Brill and J. A. Wheeler, "Interaction of neutrinos and gravitational fields," Rev. Mod. Phys. 29, 465 (1957).

[14] R. Ruffini and S. Bonazzola, "Systems of selfgravitating particles in general relativity and the concept of an equation of state," Phys. Rev. 187, 1767 (1969).

[15] Y. K. Gambhir, P. Ring and A. Thimet, "Relativistic mean field theory for finite nuclei," Annals Phys. 198 (1990) 132.

P. G. Reinhard, "The relativistic mean-field description of nuclei and nuclear dynamics," Rep. Prog. Phys. 52 (1989) 439.

[16] A. Bachelot, "The Dirac system on the Anti-de Sitter Universe," Commun. Math. Phys. 283, 127 (2008) [arXiv:0706.1315 [math-ph]].

[17] P. Horava and C. M. Melby-Thompson, "Anisotropic Conformal Infinity," arXiv:0909.3841 [hep-th]. 
[18] A. Adams, A. Maloney, A. Sinha and S. E. Vazquez, "1/N Effects in Non-Relativistic Gauge-Gravity Duality," JHEP 0903, 097 (2009) [arXiv:0812.0166 [hep-th]].

[19] See, for example:

N. D. Birrell and P. C. W. Davies, Quantum Fields In Curved Space, (Cambridge University Press, 1982).

[20] J. de Boer, K. Papadodimas and E. Verlinde, "Holographic Neutron Stars," arXiv:0907.2695 [hep-th].

[21] G. Clement, 'Spinning charged BTZ black holes and and self-dual particle like solutions", [arXiv:gr-qc/9510025].

[22] K. A. Moussa, G. Clement, H. Guennoune and C. Leygnac, "Three-dimensional ChernSimons black holes," Phys. Rev. D 78, 064065 (2008) [arXiv:0807.4241 [gr-qc]].

[23] T. Andrade, M. Banados, R. Benguria and A. Gomberoff, "The $2+1$ charged black hole in topologically massive electrodynamics," Phys. Rev. Lett. 95, 021102 (2005) [arXiv:hepth/0503095].

[24] A. Niemi and G. Semenoff, "Axial-anomaly-induced fermion fractionization and effective gauge-theory actions in odd-dimensional space-times", Phys. Rev. Lett. 512077 (1983).

[25] M. Banados, C. Teitelboim and J. Zanelli, "The Black hole in three-dimensional spacetime," Phys. Rev. Lett. 69, 1849 (1992) [arXiv:hep-th/9204099].

[26] G. Clement, "Classical solutions in three-dimensional Einstein-Maxwell cosmological gravity," Class. Quant. Grav. 10, L49 (1993).

[27] C. Martinez, C. Teitelboim and J. Zanelli, "Charged rotating black hole in three spacetime dimensions," Phys. Rev. D 61, 104013 (2000) [arXiv:hep-th/9912259].

[28] J. Kapusta and C. Gale, "Finite-Temperature Field Theory Principles and Applications," Cambridge Monographs on Mathematical Physics, (2006). 